Revista de Matemática: Teoría y Aplicaciones 2006 13(1) : 41-51

CIMPA - UCR - CCSS ISSN: 1409-2433

\title{
MMDS AND 2D SCATTERING PROBLEM BY CYLINDRICAL STRUCTURE WITH PIECE-WISE SMOOTH BOUNDARY
}

\author{
Alexander P. Anyutin* $\quad$ V. I. Stasevich ${ }^{\dagger}$ \\ Recibido/Received: $21 \mathrm{Feb} 2004$. Aceptado/Accepted: 15 Dec 2005
}

\begin{abstract}
An universal modification of the method of discrete sources (MMDS) was applied for solving 2D Dirichlet or Neumann boundary problem when the scatterer's contour is a piece-wise smooth contour. The problems of accuracy, choosing auxiliary contours, stable results, location and type of contour's break points are discussed.
\end{abstract}

Keywords: Modification of the method of discrete sources, piece-wise smooth boundary, accuracy.

\section{Resumen}

Una modificación universal al método de fuentes discretas (MMDS) ha sido aplicada para resolver problemas 2D de frontera de Dirichlet o Neumann cuando el contorno del dispersador es un contorno suave a trazos. Se discuten los problemas de precisión, escogiendo contornos auxiliares, resultados estables, localización y tipo de contorno en los puntos de quiebre.

Palabras clave: Modificación del método de fuentes discretas, frontera suave a trozos, precisión.

Mathematics Subject Classification: 34L25, 74S15, 65R20.

\footnotetext{
${ }^{*}$ Russian New University, 22, Radio Street, Moscow 105007, Russia. Tel: +(095)182-2624, E-Mail: anioutine@mail.ru

${ }^{\dagger}$ Russian New University 60-2-94, Novocheremushkinskaya UI 117420 Moscow, Russia. E-Mail: walter@robis.ru
} 


\section{Introduction}

In recent years it is seen that such method as the method of discrete sources (MDS) [1] is widely practiced due to their simplicity. One could find too that MDS had three stages in its developing. The first one deal with work [1] in which had been proved the completeness for a set of fundamental solutions (auxiliary sources) to the 2D (or 3D) Helmholtz equation (the vector case was considered too). These fundamental solutions have singularities on a certain closed auxiliary surface $\Sigma$ (or auxiliary contour $\Sigma$ ) inside of the scatter but theorems had been proved by V.D. Kupradze [1] places almost no constraints on the geometry of the auxiliary surface $\Sigma$ (auxiliary contour $\Sigma$ ). The second stage was connected with numerous publications in which MDS has been applied for numerical solution of many scattering and diffraction problems. However, only one essential scientific result was achieved in this period - it was detected the fact of unstable algorithms and decreasing accuracy with increasing the number of auxiliary sources-. In works [2,3,4], A.G.Kyurlchan found the origin of this effect and constraints on the geometry of $\Sigma$ were formulated in the form of the theorem. According with his theorem an auxiliary contour $\Sigma$ have to enclose all of the singularities of the diffracted field inside of the scatter's surface (contour) $S$. Nevertheless almost all published works [ 5,6 and references in them] do not satisfy to condition of this theorem and its results could not be considered as correct one. But that theorem also did not show the way of constructing geometry of auxiliary surface $\Sigma$ (or auxiliary contour $\Sigma$ ) and its location. So, the third stage was made in $[7,8]$ where the way of the constructing for MDS was appointed and named as universal modification of the method of discrete sources (MMDS). But both MDS and its universal modification MMDS have one essential restriction - the surface (cross-section contour) of the scatterer has to be described by analytical function only-.

This paper is concerned the extending MMDS technique for solving a scattering problem by $2 \mathrm{D}$ scatterer with piece-wise smooth contour. The problem of choosing the auxiliary surfaces, accuracy and types of the contour's break points were considered too.

\section{2D Scattering Problem}

At first let us consider a scattering of $E$ polarized wave $u_{0}(\vec{r})$ by perfect conducting cylinder with cross section contour $S$ :

$$
\begin{aligned}
\rho(\varphi) & =r_{1}(\varphi) \text { for } \varphi_{1} \leq \varphi \leq-\varphi_{1}, \\
\rho(\varphi) & =r_{2}(\varphi) \text { for } \varphi \notin\left[-\varphi_{1}, \varphi_{1}\right], \\
r_{1}\left( \pm \varphi_{1}\right) & =r_{2}\left( \pm \varphi_{1}\right),
\end{aligned}
$$

in cylindrical system of coordinates $(z, r, \varphi), r_{1}(\varphi), r_{2}(\varphi)$ functions describing two parts of the contour $S$ (see Fig.1a).

Diffracted field $u^{1}(\vec{r})$ is a solution of the Helmholts equation

$$
\Delta u^{1}+k^{2} u^{1}=0
$$


without of $S$ and satisfies to Dirichlet boundary condition ( $E$ polarized incident wave) on $S$

$$
\left.\left.u(\vec{r})\right|_{S} \equiv\left[u_{0}(\vec{r})+u^{1}(\vec{r})\right]\right|_{S}=0
$$

or Neumann boundary condition (H polarized incident wave) on $S$

$$
\left.\left.\frac{d}{d n} u(\vec{r})\right|_{S} \equiv \frac{d}{d n}\left[u_{0}(\vec{r})+u^{1}(\vec{r})\right]\right|_{S}=0
$$

and Sommerfeld radiation condition

$$
\frac{\partial u^{1}(\vec{r})}{\partial r}+i k u^{1}(\vec{r})=o\left(r^{-1 / 2}\right),|r| \rightarrow \infty
$$

where $k$ is a wave number of the free space.

According to the MMDS, the diffracted field $u^{1}(\vec{r})$ outside of $S$ could be presented as follows

$$
u^{1}(r, \varphi)=\sum_{m=1}^{M} A_{m} H_{0}^{(2)}\left(k\left|\vec{r}-\vec{r}_{m}\right|\right) .
$$

Here, $H_{0}^{(2)}\left(k\left|\vec{r}-\vec{r}_{m}\right|\right)$ is the fundamental solution to the Helmholz equation (auxiliary source); $A_{m}$ are the coefficients to be determined; $\left|\vec{r}-\vec{r}_{m}\right|=\left[r^{2}+r_{m}^{2}-2 r r_{m} \cos \left(\varphi-\varphi_{m}\right)\right]^{1 / 2}$ is the distance between points given by the radius vectors $\vec{r}$ and $\vec{r}_{m}$ in polar coordinates; $\vec{r}_{m}$ are the radius vectors positions of the sources on auxiliary contour $\Sigma ; M$ is the total number of auxiliary sources on $\Sigma$. Placing $\vec{r}$ on $S$ and satisfying (3) in $M$ points we could obtain a system of $M$ linear algebraic equations for unknown coefficients $A_{m}$.

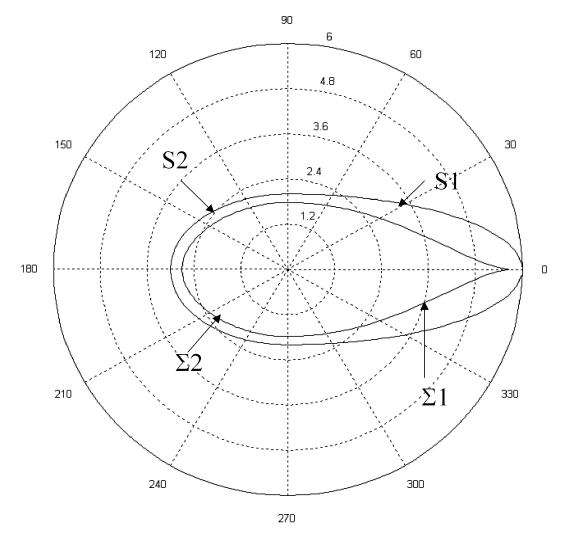

Figure 1:(a): Structure of the original $S_{1}, S_{2}$ contours and auxiliary $\Sigma_{1}, \Sigma_{2}$ contours for (7) and $\varphi_{1}=\pi / 2$.

As function $\rho(\varphi)$ for total contour $S$ consists of two smooth functions $r_{1}(\varphi), r_{2}(\varphi)$ accordingly and presented by (1) then we will construct a total auxiliary contour $\Sigma$ as a contour consisting of two auxiliary contours $\Sigma_{1}$ and $\Sigma_{2}$ (see Fig.1a). For each auxiliary 
contour $\Sigma_{1}$ (we consider that it has equation $r_{\Sigma_{1}}(\theta)$ ) and $\Sigma_{2}$ (we consider that it has equation $r_{\Sigma_{2}}(\theta)$ ) we construct auxiliary contours $r_{\Sigma_{1}}(\varphi), r_{\Sigma_{2}}(\varphi)$ accordingly by analytical transformation of the each original contours $r_{1}(\varphi), r_{2}(\varphi)[2-4,7,8]$ as follows:

$$
\begin{aligned}
\zeta_{1} & =r_{1}(\varphi) \exp \{i \varphi\} ; \varphi=\varphi^{\prime}+i \varphi_{1}^{\prime \prime} ; \\
r_{\Sigma_{1}}(\theta) & =\left|\zeta_{1}\right| ; \theta=\arg \zeta_{1} \text { for }-\varphi_{1} \leq \varphi^{\prime} \leq \varphi_{1}
\end{aligned}
$$

for $\Sigma_{1}$ contour, and

$$
\begin{aligned}
\zeta_{1} & =r_{2}(\varphi) \exp \{i \varphi\} ; \varphi=\varphi^{\prime}+i \varphi_{1}^{\prime \prime} ; \\
r_{\Sigma_{2}}(\theta) & =\left|\zeta_{2}\right| ; \theta=\arg \zeta_{2} \text { for other } \varphi^{\prime}
\end{aligned}
$$

for $\Sigma_{2}$ contour.

In case of two original contours $r_{1}(\varphi), r_{2}(\varphi)$ as ellipses (see Fig.1a)

$$
\begin{aligned}
& r_{1}(\varphi)=a_{1} / \sqrt{1-\varepsilon_{1}^{2} \cos ^{2}(\varphi)} ; \varepsilon_{1}^{2}=1-\left(a_{1} / b_{1}\right)^{2} ; \\
& r_{2}(\varphi)=a_{1} / \sqrt{1-\varepsilon_{2}^{2} \cos ^{2}(\varphi)} ; \varepsilon_{2}^{2}=1-\left(a_{1} / b_{2}\right)^{2} ;
\end{aligned}
$$

or ellipse and rectilinear line (see Fig.1b)

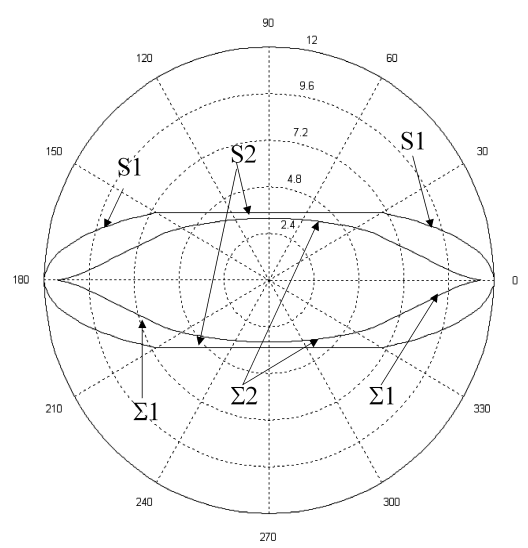

Figure 1:(b): Structure of the original $S_{1}, S_{2}$ contours and auxiliary $\Sigma_{1}, \Sigma_{2}$ contours for (8) and $\varphi_{1}=\pi / 6$.

$$
\begin{aligned}
r_{1}(\varphi) & =a_{1} / \sqrt{1-\varepsilon_{1}^{2} \cos ^{2}(\varphi)} ; \varepsilon_{1}^{2}=1-\left(a_{1} / b_{1}\right)^{2} ; \text { for } \\
-\varphi_{1} & \leq \varphi \leq \varphi_{1} \text { and } \pi-\varphi_{1} \leq \varphi \leq \pi+\varphi_{1} \\
r_{2}(\varphi) & =r_{1}\left(\varphi_{1}\right) / \sin (\varphi) \text { for } \pi-\varphi_{1} \leq \varphi \leq \varphi_{1}, \text { and } \\
\pi+\varphi_{1} & \leq \varphi \leq 3 \pi / 2+\varphi_{1}
\end{aligned}
$$

or ellipse $r_{1}(\varphi)(8)$ and $r_{2}(\varphi)$ as multifoil

$$
r_{2}(\varphi)=a+b \cos (q \varphi), \quad q=2,3, \ldots
$$


one could obtain an analytical expression for $\varphi_{1,2}^{\prime \prime}=\varphi_{q 1,2}^{\prime \prime}-\delta$ (where $\varphi_{q 1,2}^{\prime \prime}$ determinates the location of auxiliary contour $\Sigma_{q 1,2}$ which is passed through singularities, $\delta$ is a parameter showing difference between $\Sigma_{q 1,2}$ and $\Sigma$ contours) [5-8]. If $r_{i}(\varphi)$ is arbitrary analytical function then value $\varphi_{q i}^{\prime \prime}$ could be found by numerically $[9,10]$.

We would like to remind that total auxiliary contour $\Sigma$ have to enclose all principal singularities and only in this case we will have a unique solution of scattering problem [2-4]. These singularities consists of singular points of the analytical extension of the function $\left.u_{0}(\vec{r})\right|_{S}$ to the region inside $S$, points at which one-to-one correspondence (6) or (7) is violated satisfying the relationship

$$
\zeta^{\prime}=\left[\rho^{\prime}(\varphi)+i \rho(\varphi)\right] \exp (i \varphi)=0,
$$

the imaginary source on physical list of the Riemann surface (in case of cylindrical incidence wave) and break points of total original contour $\rho(\varphi)$. The influence of the imaginary source's location and singular points (11) on accuracy was investigated in [7-10]. In this paper we explored the influence of the type of breakpoints for total original contour's function $\rho(\varphi)$ on accuracy problem.

Some examples of constructed original $S$ and auxiliary contours $\Sigma$ are presented at Fig. 1a and Fig.1b, respectively. These contours were under consideration when the scattering pattern was calculated and accuracy problem was explored. We would like to stress the difference between constructing auxiliary contours $\Sigma_{1}, \Sigma_{2}$ in Fig.1b and Fig.1c. In the first case (Fig.1b) we make an analytical transformation of the original contour but for second one (Fig.1c) we use a simple straight line $\left(\Sigma_{2}\right)$ for connection of the end's points of $\Sigma_{1}$. Such type of approximation is often used in practice.

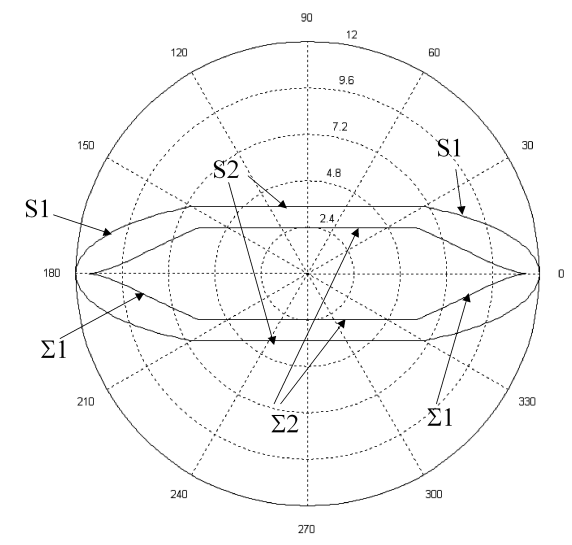

Figure 1.(c): Structure of the original $S_{1}, S_{2}$ contours and auxiliary $\Sigma_{1}, \Sigma_{2}$ contours for (8) and $\varphi_{1}=\pi / 6$.

The accuracy of the solving problems we estimate as the residual $\Delta=\left|u_{0}\left(\vec{r}_{S}\right)-u^{1}\left(\vec{r}_{S}\right)\right|$ of the boundary conditions in the middle point for each $m$-interval on $S$ :

$$
\varphi_{m+1}-\varphi_{m} ; \quad m=1, \ldots, M ; \quad \varphi_{m}=2 m \pi / M
$$




\section{Discussion of the results}

First of all we had explored the scattering of the plane incident wave

$$
u_{0}=\exp \left\{-i k r \cos \left(\varphi-\varphi_{0}\right)\right\}
$$

by elliptical cylindrical structure (8) with $\varphi_{1}=\pi / 2$. In this case we had a situation when function $\rho(\varphi)(1)$ is continuous $\left(r_{1}\left( \pm \varphi_{1}\right)=r_{2}\left( \pm \varphi_{1}\right)\right)$ and its deviation $\left(d r_{1}\left( \pm \varphi_{1}\right) / d \varphi=\right.$ $\left.d r_{2}\left( \pm \varphi_{2}\right) / d \varphi=0\right)$ is continuous too (see Fig. 1a). Elliptical structure (8) had parameters: $k a_{1}=4, k b_{1}=12, k a_{2}=4, k b_{2}=6(k D \approx 18 ; D$ is a maximum size of the scatterer's domain) or when $k a_{1}=20, k b_{1}=60, k a_{2}=20, k b_{2}=30(k D \approx 90 \gg 1)$; angle of incidence $\varphi_{0}$ was changed from 0 up to $\pi ; M=256$ and $\delta=10^{-6}$. By the way, we had for residual's value $\max (\Delta) \leq 1.2 \times 10^{-11}$ in case of single ellipses $\left(r_{1}(\varphi)\right.$ or $\left.r_{2}(\varphi)\right)$ with $M=256$ and $\delta=10^{-6}$. It was detected that residual $\Delta$ practically does not depend both on the angle $\varphi_{0}$ and the value $k D$. A typical figure of $\Delta$ is presented at Fig. 2. It is seen that $\Delta$ has two rather narrow maximums in the neighbor of the breakpoints of $S(\Sigma)$ and we have a total reducing of the accuracy for all points of the contour $S$. But at any rate we have following estimation $\log (\Delta) \leq-5$ for all points on $S$.

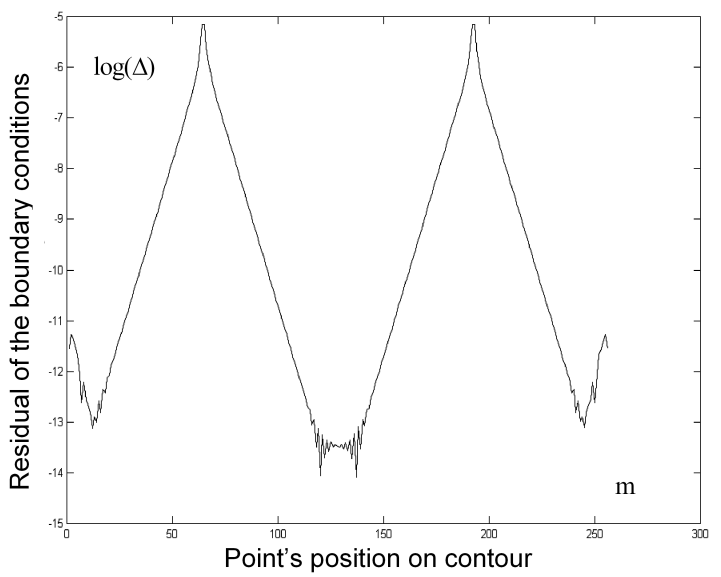

Figure 2: The residual for two ellipses' structure (8) and $E$ polarized plane incident wave and $\varphi_{0}=0$.

The relative amplitude of the scattering pattern for two ellipses' structure (8) with parameters: $k a_{1}=4, k b_{1}=12, k a_{2}=4, k b_{2}=6(k D=18) M=256, \delta=10^{-6}$ and $E$ polarized plane incident wave with $\varphi_{0}=\pi / 2$ is presented by Fig.3.

The residual $\Delta$ for cylindrical structure (8), $k a_{1}=20, k b_{1}=60, k a_{2}=20, k b_{2}=30$ $(k D=90) M=512, \delta=10^{-6}$ and $H$ polarized plane incident wave with $\varphi_{0}=\pi / 2$ presented at Fig.4. It is seen that residual $\Delta$ has just the same view as the curve at Fig.2 but we have some worse value $\Delta$ then for a case of $E$ polarized plane incident wave. The relative amplitude of the scattering pattern for this case is shown at Fig.5.

The residual $\Delta$ for of cylindrical structure (9) with parameters: $k a_{1}=4, k b_{1}=12$, $\varphi_{1}=\pi / 6, \varphi_{0}=0, M=512, \delta=10^{-6}$ is presented at Fig. 6 . In this case we see that residual 


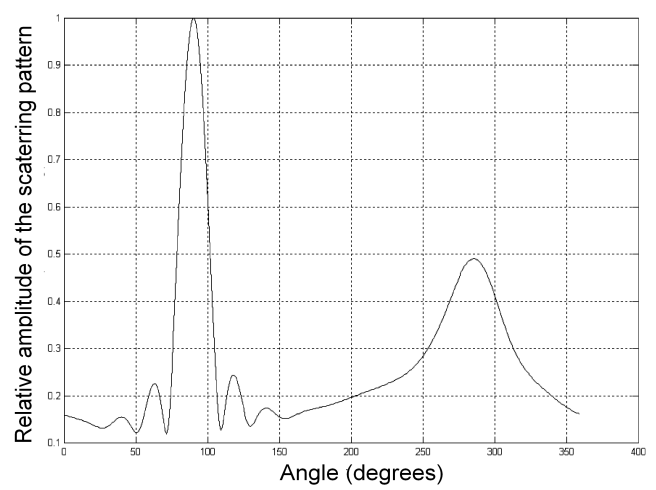

Figure 3: The relative amplitude of the scattering pattern for two ellipses' structure (8) and $H$ polarized plane incident wave $(k D \approx 18)$.

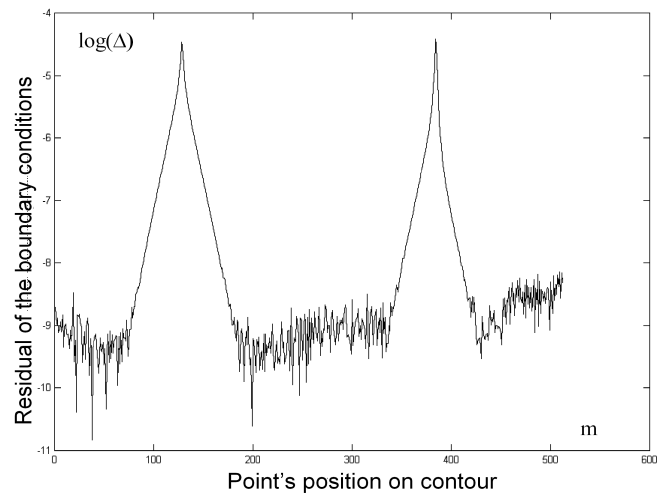

Figure 4: The residual for two ellipses' structure (8) and $H$ polarized plane incident wave $(k D \approx 90)$.

$\Delta$ have four maximums in neighbor of the breakpoints $S(\Sigma)$ and we have a reducing of total accuracy $\left(\min (\Delta) \approx 10^{-7}\right.$ and $\max (\Delta) \approx 10^{-3}$ ) for all points on contour $S$.

Other situation takes place when we use a simple straight-line approximation (for auxiliary contour $\Sigma_{2}$ ) for connection of the end's points of auxiliary contours $\Sigma_{1}$. This type of approximation is often using in practice. Having made a substitution of auxiliary contour $\Sigma_{2}$ by rectilinear line we gets a structure presented at Fig. 1c. It is characterized by the existence of four breakpoints for $S$ and $\Sigma$ contours.

The residual's distribution $\Delta$ on $S$ for this situation is shown at Fig.7 and relative amplitude of the scattering pattern at Fig.8 respectively. One could see from Fig.7 that the value $\max (\Delta) \leq 10^{-2}$ for all points on $S$. So, accuracy situation is much worse then for Fig. 2,4,6 when an analytical transformation of original contour was made.

¿From Fig.8 one can see that the relative amplitude of the scattering pattern has oscillations in $\pi<\varphi<2 \pi$ region (it is shadow region for incident wave). As we are in $k D \gg 1$ region then it could be explained as follows. It is known that breakpoint of $S$ radiate a diffraction wave (or Keller's diffracted edge rays of Geometrical theory of 


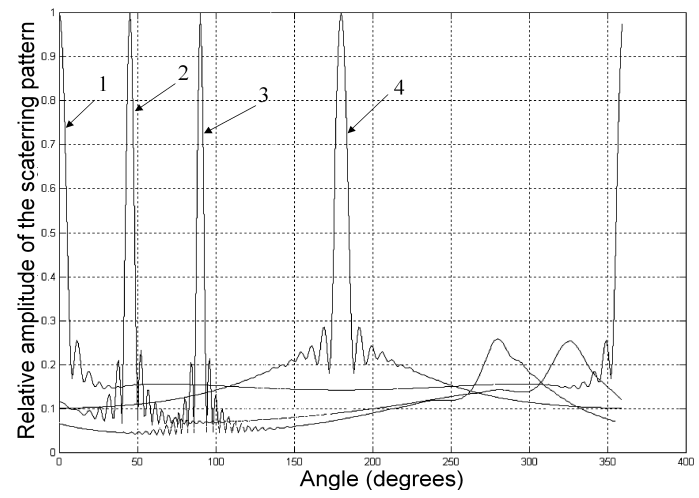

Figure 5: The relative amplitude of the scattering pattern for two ellipses structure (8) and $H$ polarized plane incident wave $(k D \approx 90 \gg 1) ; 1-\varphi_{0}=0,2-\varphi_{0}=\pi / 4,3-\varphi_{0}=\pi / 2$, $4-\varphi_{0}=\pi$.

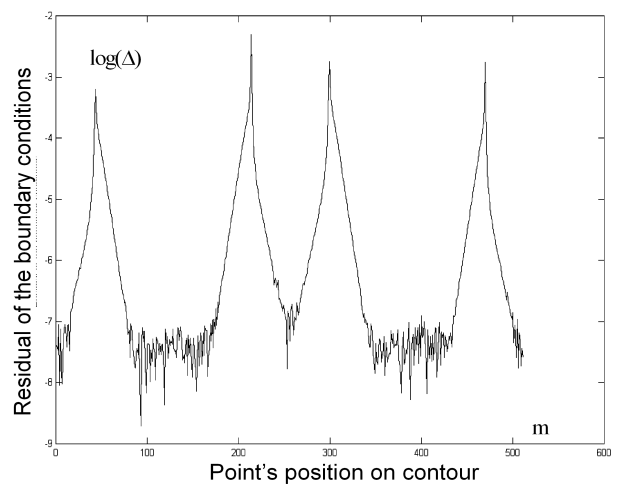

Figure 6: The residual for ellipse-line structure (9) Fig.1b and $E$ polarized plane incident wave.

diffraction $[11,12])$. In our case we have situation when two breakpoints of $S$ are located both in light and shadow region of $S$ respectively. In light region amplitude of diffracted edge rays are small with regard to the amplitude of the geometrical optic rays. In shadow region the geometrical optic rays are absent. So in this region we have interaction of the fields for diffracted edge and gliding rays as their amplitudes are commensurably.

At the end of this part we consider situation when total contour $S$ consists of ellipse (8) (with parameters $k a=10 ; k b=40$ ) and multifoil (10) (with parameters $k a=9$; $k b=1 ; q=24)$. The structure of original and auxiliary contours is shown at Fig.9. Fig.10 illustrates the relative amplitude of the scattering pattern for plane incident wave with angle of incidences $\varphi_{0}=0$ and $\varphi_{0}=\pi$ when $M=800$ and $\delta=10^{-6}$.

It stands to reason that presented results show that accuracy situation of MMDS became worse in case of existing the break points for original contour $S$. Nevertheless, the accuracy is rather high when functions $r_{\Sigma}(\theta)$ and $d r_{\Sigma}(\theta) / d \theta$ for total auxiliary contour $\Sigma$ are continuous. Otherwise we will have a bad situation for accuracy. It is clear that 


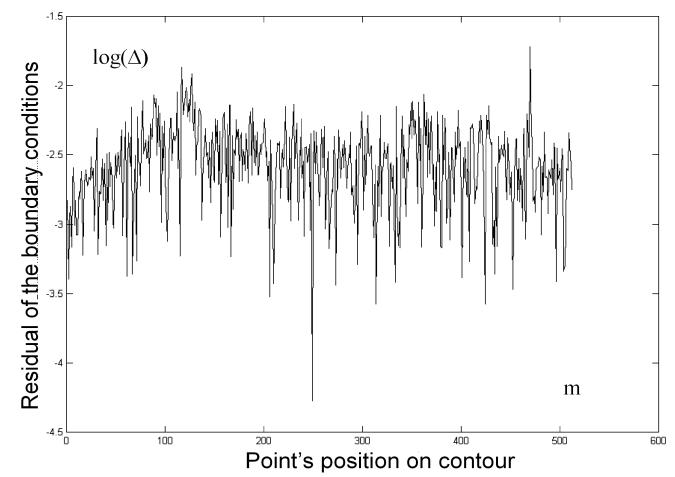

Figure 7: The residual for ellipse-line structure (9) Fig.1c and $E$ polarized plane incident wave.

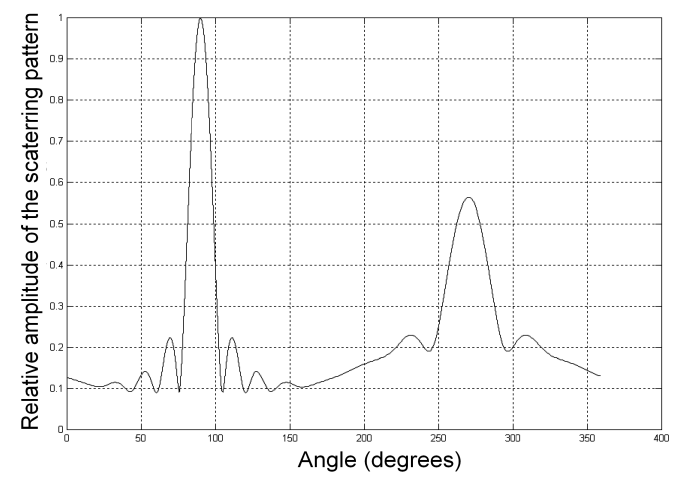

Figure 8: The relative amplitude of the scattering pattern for ellipses-line structure Fig.1c and $E$ polarized plane incident wave with $\varphi_{0}=\pi / 2$.

procedure described above could be applied for approximation an arbitrary contour $S$ as it could be presented by pieces of ellipse, multifoil and straight lines.

\section{Conclusion}

The developed results show that MMDS could be applied directly for solving a scattering problem by scatterer with piece-wise smooth contours and a high accuracy could be achieved. The method can easily be extended to 3D case and vector fields.

\section{Acknowledgments}

This work was supported by the Russian Foundation for Basic Research, project No. 03-02-16336. 


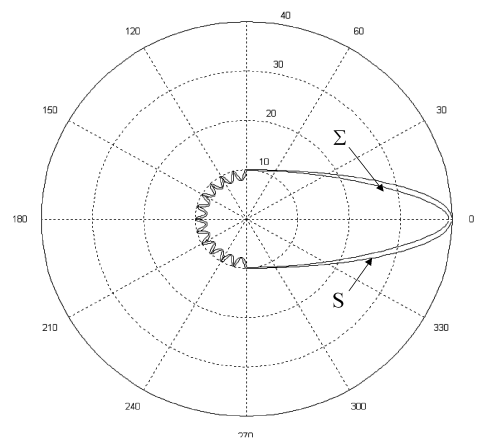

Figure 9: Structure of the original contours and auxiliary contours $\Sigma$ for ellipse-multifoil cylindrical structure (10).

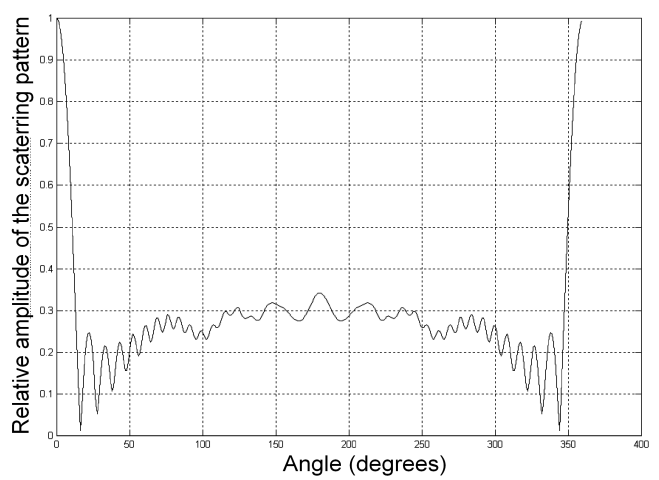

Figure 10: The relative amplitude of the scattering pattern for ellipse-multifoil structure (10) and $H$ polarized plane incident wave with $\varphi_{0}=0$.

\section{References}

[1] Kupradze, V.D. (1967) "On the approximate solutions of problems in mathematical physics", Journal Success Mathematical Sci. 22: 58-108.

[2] Kyurkchan, A.G. (1986) "Analytical continuation of wave fields", Journal of $R a$ diotekh. and Electron 31: 1294-1303.

[3] Kyurkchan, A.G. (1986) "Representation of diffraction fields by wave potentials and the method of auxiliary currents in problems of the diffraction of electromagnetic waves", Journal Radiotekh. and Electron 31: 20-27.

[4] Kyurkchan, A.G.; Sternin, B.Yu.; Shatalov, V.E. (1996) "Singularities of continuation of wave fields", Journal Usp. Fiz. Nauk 166: 1285-1312. 
[5] Obelleiro, F.; Landesa, L.; Rodrigues, J.L.; Pino, M.R.; Sabariego, R.V.; Leviatan, Y. (2001) "Localized iterative generalized multipole technique for large two-dimentional scattering problems" IEEE Tr. on Antennas and Propagation 49: 961-970.

[6] Kaklamani, D.I.; Anastassiu, H.T. (2002) "Aspects of the method of auxiliary sources in computational electromagnetics", IEEE Antenna and Propagation Magazine 44: $48-64$.

[7] Kyurkchan, A.G.; Minaev, S.A.; Soloveichik, A.L. (2001) "A modification of the method of discrete sources based on prior information about singularities of the diffracted field", Journal of Radiotekh. and Electron 46: 666-672.

[8] Anyutin, A.P.; Kyurkchan, A.G.; Minaev, S.A. (2002) "A modification of the method of discrete sources", Journal Radiotekh. and Electron 47(8): 955-960.

[9] Anyutin, A.P.; Kyurkchan, A.G.; Minaev, S.A. (2002) "Application of MMDS in 2D and 3D scattering problems", Proceedings of International Symposium on Antennas JINA 2002 (Vol.2), Nice : 213-216.

[10] Anyutin, A.P.; Kyurkchan, A.G.; Minaev, S.A. (2002) "Universal modification of the 2D and 3D method of discrete sources", Proceedings of XXVII-th URSI General Assembly, Maastricht, August.

[11] Keller, J.B. (1962) "Geometrical theory of diffraction", Journal of Opt. Soc. Amer. 52(2): 116-130.

[12] Borovikov, V.A.; Kinber, B.E. (1978) Geometrical Theory of Diffraction. Svyaz, Moscow. 